\title{
High-affinity binding site for a group II intron-encoded reverse transcriptase/maturase within a stem-loop structure in the intron RNA
}

\author{
KAZUO WATANABE and ALAN M. LAMBOWITZ
}

Institute for Cellular and Molecular Biology, Department of Chemistry and Biochemistry, and Section of Molecular Genetics and Microbiology, School of Biological Sciences, University of Texas at Austin, Austin, Texas 78712, USA

\begin{abstract}
Mobile group II introns encode proteins that have reverse transcriptase and maturase activities and bind specifically to the intron RNA to promote both RNA splicing and intron mobility. Previous studies with the Lactococcus lactis LI.LtrB intron showed that the intron-encoded protein (LtrA) has a high-affinity binding site in intron subdomain DIVa, an idiosyncratic structure containing the translation initiation region of the LtrA open reading frame, and that this binding site consists of a small stem-loop emanating from a purine-rich internal loop. The binding of LtrA to DIVa is important for translational regulation, RNA splicing, and intron mobility. Here, we show by in vitro selection that part of the purine-rich internal loop can be closed by base pairing, enabling the LtrA binding site to be represented as an extended stem-loop structure with a bulged A (A556) required for tight binding of LtrA. The deletion or pairing of A556 has relatively little effect on maturase-promoted RNA splicing, but significantly inhibits intron mobility. The wild-type DIVa structure has a second bulged A (A553), which is selected against in tightly binding variants. As expected from the selection, the deletion or pairing of A553 results in tighter binding of LtrA, but surprisingly, also inhibits intron mobility. These findings suggest that the binding of LtrA to DIVa is delicately balanced, so that either too weak or too tight binding can be deleterious. The nature of the maturase/DIVa interaction and its role in translational regulation are reminiscent of the coat protein/RNA hairpin interactions of single-stranded RNA phages.
\end{abstract}

Keywords: ribozyme; RNA-protein interaction; RNA structure; translational regulation

\section{INTRODUCTION}

Mobile group II introns encode reverse transcriptases (RTs) that function in intron mobility and also promote RNA splicing by stabilizing the catalytically active structure of the intron RNA (maturase activity; Lambowitz et al. 1999; Belfort et al. 2002; Lambowitz and Zimmerly 2004). The best characterized mobile group II introns are the yeast mtDNA introns aI 1 and $\mathrm{aI} 2$ and the Lactococcus lactis Ll.LtrB intron. Studies with these introns showed that the intron-encoded protein (IEP) binds specifically to the intron in unspliced precursor RNA to promote RNA splicing, and then remains bound to the excised intron lariat RNA in a ribonucleopro-

Reprint requests to: Alan M. Lambowitz, Institute for Cellular and Molecular Biology, Department of Chemistry and Biochemistry, and Section of Molecular Genetics and Microbiology, School of Biological Sciences, University of Texas at Austin, Austin, TX 78712, USA; e-mail: lambowitz@mail.utexas.edu; fax: (512) 232-3420.

Article published online ahead of print. Article and publication date are at http://www.rnajournal.org/cgi/doi/10.1261/rna.7730104. tein (RNP) particle that mediates intron mobility (Zimmerly et al. 1995a,b; Yang et al. 1996; Matsuura et al. 1997; Saldanha et al. 1999). RNPs initiate mobility by binding to DNA and recognizing a 30-35-bp DNA target site, with both the IEP and base pairing of the intron RNA contributing to DNA target site recognition. The intron RNA then reverse splices directly into one strand of the duplex DNA, while the IEP cleaves the opposite strand and uses the cleaved $3^{\prime}$ end as a primer for reverse transcription of the reverse spliced intron RNA. Genomic integration of the resulting cDNA occurs via host cell DNA recombination or repair pathways, which can differ in different hosts (Eskes et al. 1997, 2000; Cousineau et al. 1998).

The binding of the IEP to the intron RNA is critical for both RNA splicing and intron mobility. Previous studies with the L. lactis Ll.LtrB intron suggested a model in which its IEP, denoted LtrA protein, binds first to a high-affinity binding site in intron subdomain DIVa, an idiosyncratic structure that lies outside the catalytic core and contains the ribosome binding site and initiation codon of the LtrA open 
reading frame (ORF; Wank et al. 1999). Experiments using reporter gene constructs showed that the binding of LtrA to DIVa down-regulates translation, thereby preventing the accumulation of excess IEP and halting ribosome entry into the intron, which might otherwise impede RNA splicing (Singh et al. 2002). After binding to DIVa, LtrA makes weaker secondary contacts with conserved catalytic core regions to stabilize the active structure for RNA splicing and then remains associated with the excised intron RNA, presumably using some or all of the same interactions, to constitute the RNPs that function in intron mobility (Wank et al. 1999; Matsuura et al. 2001). RNA footprinting and structure mapping experiments identified potential secondary binding sites in DI, DII, and DVI and demonstrated that binding of LtrA stabilizes conserved tertiary structure required for catalytic activity (Matsuura et al. 2001).

Although DIVa is required for maximal RNA splicing, an Ll.LtrB intron deleted for DIVa still shows some residual maturase-dependent RNA splicing, which occurs by the direct binding of the IEP to the catalytic core (Wank et al. 1999; Matsuura et al. 2001; Cui et al. 2004). DIVa contributes to RNA splicing by increasing the binding affinity for the maturase by $\sim 10^{5}$, and the binding of the maturase to DIVa also appears to facilitate RNA structural transitions that are rate limiting for splicing at saturating protein concentrations (Wank et al. 1999; Matsuura et al. 2001). Additionally, binding to DIVa helps position the IEP to initiate cDNA synthesis just downstream from the intron in the $3^{\prime}$ exon, as required for intron mobility (Wank et al. 1999).

The yeast aI2 maturase also has a high-affinity binding site in DIVa, which is required for the stable binding of its IEP in vivo (Huang et al. 2003). This finding is significant because the yeast aI 2 maturase is translated as a preprotein with the upstream exons, and DIVa is no longer used for translation initiation, thus separating its role in that process from its other critical functions. The deletion of DIVa from the yeast aI2 intron inhibited RNA splicing, but the level of residual splicing in vivo in the absence of DIVa was considerably higher than that for the Ll.LtrB intron (70\% and 6\%-10\%, respectively; Huang et al. 2003; Cui et al. 2004). In both the yeast and lactococcal introns, however, the deletion of DIVa almost completely abolished intron mobility, suggesting that the DIVa contacts are more critical for that process than they are for RNA splicing (D’Souza and Zhong 2002; Huang et al. 2003).

The structure of the DIVa binding site in the Ll.LtrB intron was investigated previously by in vitro selection and mutagenesis (Singh et al. 2002). These experiments used a small (119-nt) RNA consisting of DIVa plus the DIV stem loop (denoted DIV+IVa RNA), with nucleotide residues 537-598 "doped" 30\% (i.e., synthesized with 10\% of each non-wild-type nucleotide residue at each position; Singh et al. 2002). The results showed that the LtrA binding site consists of a small terminal stem-loop (iii) emanating from a purine-rich internal loop, with both sequence and secondary structure contributing to LtrA binding (Fig. 1A). Seven nucleotide residues, shown in red in Figure 1A, were found to be invariant in tightly binding DIVa clones, in-
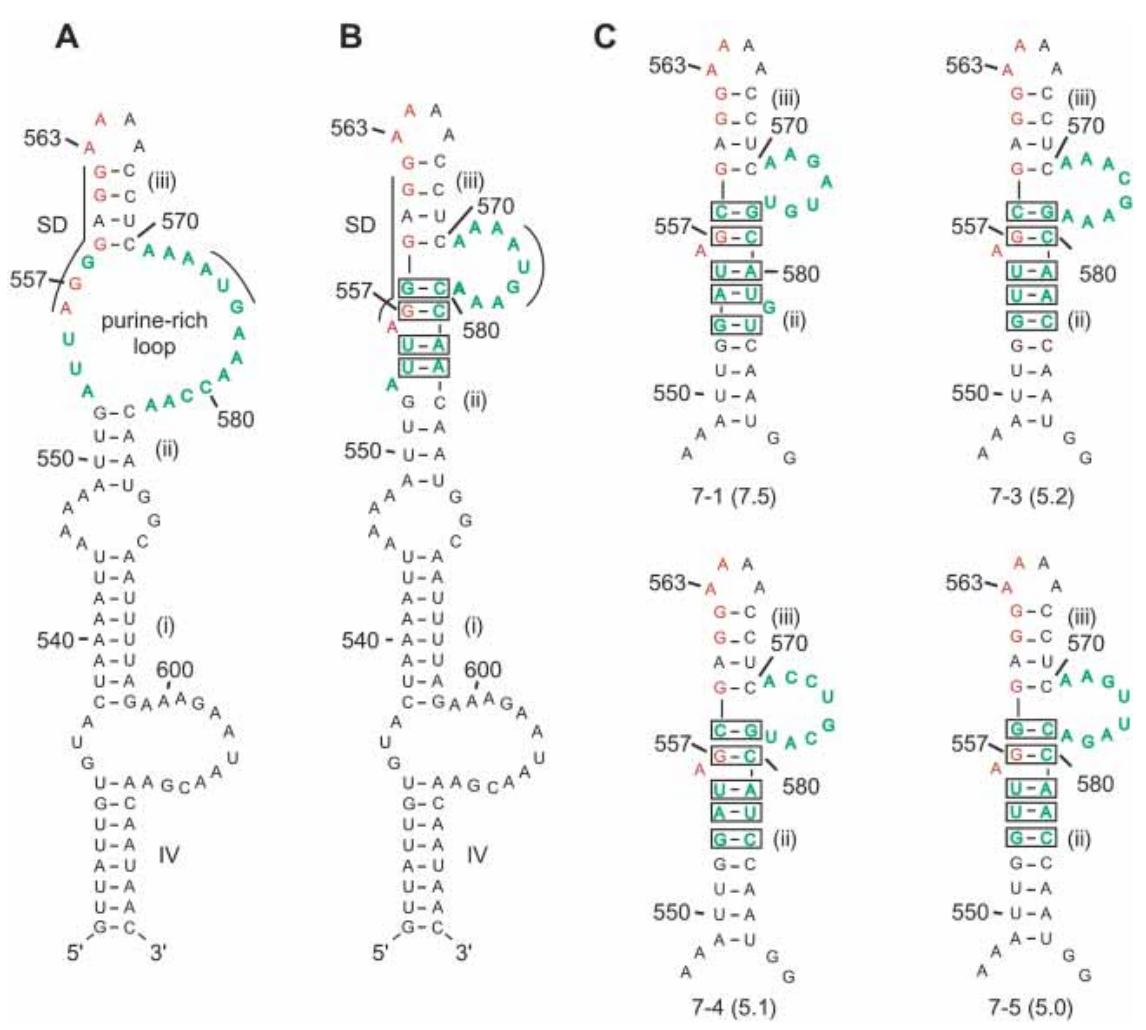

FIGURE 1. Predicted secondary structures of the wild-type DIV+IVa RNA and variants obtained after in vitro selection for LtrA binding. (A) Wild-type DIV+IVa structure based on the in vitro selection of Singh et al. (2002), in which positions 537-598 were partially randomized. (B) Wild-type DIV+IVa structure based on the in vitro selection in this work in which nucleotide residues in the purine-rich internal loop were randomized. $(C)$ Predicted secondary structures of four DIV+IVa variants obtained after seven rounds of in vitro selection for LtrA binding. Binding ratios determined by competition binding assays between variant and wildtype DIV+DIVa RNAs are indicated in parentheses. Nucleotide residues that were invariant in the in vitro selection of Singh et al. (2002) are shown in red, and those that were randomized in the in vitro selection in this work are shown in green. Base pairs confirmed by compensatory changes in the in vitro selection in this work are boxed, along with the G557:C581 pair used as the basis for alignment (see text). The previous in vitro selection confirmed the four base pairs in stem (iii) and the base pair 552:584 under the bulged A553 in stem (ii) (Singh et al. 2002). In $A$ and $B$, "SD" indicates the Shine-Dalgarno sequence of the LtrA ORF; the AUG initiation codon is delineated. 
cluding five in stem-loop (iii) and A556 and G557 in the purine-rich internal loop. The latter nucleotide residues along with those in stem (iii) are part of the predicted Shine-Dalgarno (SD) sequence for the LtrA ORF. Nucleotide substitutions for each of the invariant nucleotide residues inhibited LtrA binding, with the strongest effects found for mutations in A563 and A564 in loop (iii). The structure of the binding site suggests that LtrA binding down-regulates translation by occluding the Shine-Dalgarno sequence, either directly or by stabilizing RNA secondary structure or both (Singh et al. 2002).

Here, we carried out additional in vitro selection and mutagenesis experiments to define the structure of the purine-rich internal loop. Our results show that part of this loop forms a specific secondary structure, enabling the LtrA binding site to be represented as an extended stem-loop with the invariant A556 bulged on the $5^{\prime}$ side of the stem. The wild-type DIVa structure contains a second bulged A (A553), which antagonizes LtrA binding. The characteristics of the DIVa binding site along with its role in translational regulation are reminiscent of well-characterized coat protein/RNA hairpin interactions of single-stranded RNA phages.

\section{RESULTS}

\section{In vitro selection of partially randomized DIVa RNAs for LtrA binding}

Figure 1A shows the predicted secondary structure of DIV+IVa RNA based on the previous in vitro selection in which positions 537-598 were "doped" 30\% (Singh et al. 2002). This selection established the structure of the terminal stem-loop (iii) and identified the seven invariant nucleotide residues shown in red, but left a large purinerich internal loop (iii)/(ii), for which no secondary structure could be predicted. To investigate the structure of this internal loop, we carried out iterative in vitro selection with a pool of 136-nt DIV+IVa RNAs in which the terminal stemloop and the seven invariant nucleotide residues from the original selection were fixed, whereas the remaining $17 \mathrm{nt}$ in the purine-rich loop were randomized (indicated in green).

The progress of the selection is summarized in Table 1. In the first round, $200 \mathrm{nM}$ of the initial DIVa RNA pool (pool 0 ) containing $6 \times 10^{12}$ variants was incubated with $150 \mathrm{nM}$ of purified LtrA protein. The bound RNAs were then collected by nitrocellulose filter binding and reamplified via RT-PCR for the next round of selection. In subsequent rounds, $200 \mathrm{nM}$ RNA pool and $10 \mathrm{nM}$ LtrA protein were used for the binding reaction. The proportion of bound RNAs, monitored by ${ }^{32} \mathrm{P}$-labeled RNA retained on the filter, increased from $0.25 \%$ for pool 1 to $0.68 \%-0.75 \%$ for pools 6 and 7 , compared to $0.50 \%$ for the wild-type RNA under the same conditions. The low proportion of bound wildtype RNA is thought to reflect that the small RNA easily misfolds into an inactive structure (Wank et al. 1999).
TABLE 1. In vitro selection of DIV+IVa RNAs with randomized nucleotide residues in the purine-rich internal loop

\begin{tabular}{|c|c|c|c|c|c|}
\hline \multirow[b]{2}{*}{ Pool } & \multirow{2}{*}{$\frac{\text { Volume }}{(\mu \mathrm{l})}$} & \multirow{2}{*}{$\frac{\text { [Protein] }}{(\mathrm{nM})}$} & \multirow{2}{*}{$\frac{[\mathrm{RNA}]}{(\mathrm{nM})}$} & \multicolumn{2}{|c|}{ Bound RNA (\%) } \\
\hline & & & & $-\mathrm{LtrA}$ & $+\mathrm{Ltr} A$ \\
\hline 0 & 1000 & 150 & 200 & 0.09 & 2.7 \\
\hline 1 & 250 & 10 & 200 & 0.10 & 0.25 \\
\hline 2 & 200 & 10 & 200 & 0.10 & 0.31 \\
\hline 3 & 200 & 10 & 200 & 0.15 & 0.53 \\
\hline 4 & 200 & 10 & 200 & 0.09 & 0.47 \\
\hline 5 & 200 & 10 & 200 & 0.07 & 0.42 \\
\hline 6 & 200 & 10 & 200 & 0.09 & 0.75 \\
\hline 7 & 200 & 10 & 200 & 0.07 & 0.68 \\
\hline WT & 250 & 10 & 200 & 0.12 & 0.50 \\
\hline
\end{tabular}

In vitro selection was carried out by incubating LtrA protein with pools of DIV+DIVa RNAs containing 17 randomized nucleotide residues in the purine-rich internal loop and then isolating complexes by nitrocellulose filter binding (see Fig. 1 and Materials and Methods). The table shows the reaction volume, protein and RNA concentrations, and the percent of input RNA bound to the nitrocellulose filter in the absence (-) or presence (+) of LtrA protein for each round of the selection. A relatively high protein:RNA ratio was used in the first round to maintain diversity in the pool, and a more stringent condition (1:20 protein:RNA) was used thereafter Pool 0 refers to the initial pool before selection.
Figure 2 summarizes the sequences and binding strengths of 46 variants cloned after seven rounds of selection. Each variant has a unique sequence for the 17 randomized positions, with no insertions or deletions. Their binding ratios relative to the wild-type DIV+IVa RNA ranged from 7.5 to 0.2 , with all but three of the variants binding as well as or better than the wild-type RNA.

We noticed that all of the sequences could be aligned by base pairing the invariant $\mathrm{G} 557$, with a $\mathrm{C}$ residue located between positions 579 and 581 in the randomized region. With this alignment, all the variants could be drawn in the common secondary structure shown for the wild-type and four variant sequences in Figure 1, B and C, respectively. This secondary structure contains an extended stem (ii) with the invariant A556 bulged out of the stem, leaving a smaller bulged loop (iii)/(ii) on the opposite side. In most sequences, the alignment to maintain the G557:C base pair required reducing the size of the bulged loop (iii)/(ii) relative to the wild-type structure. In the wild-type structure, A553 is also bulged, but this feature was not conserved in most clones and in fact appears to be selected against in tightly binding variants (see below).

The predicted secondary structure of the top of stem (ii) is supported by the selection for base pairing at each position (Table 2). Thus, positions 558:580 above G557:C581 could form a Watson-Crick or wobble UG base pair in all of the variants. Further, the base pair at $558: 580$ is GC or CG in 40 of 46 variants, and the putative partner of the invariant G557 is always $\mathrm{C}$ rather than $\mathrm{U}$. As a result, most of the selected variants have two strong base pairs above the 


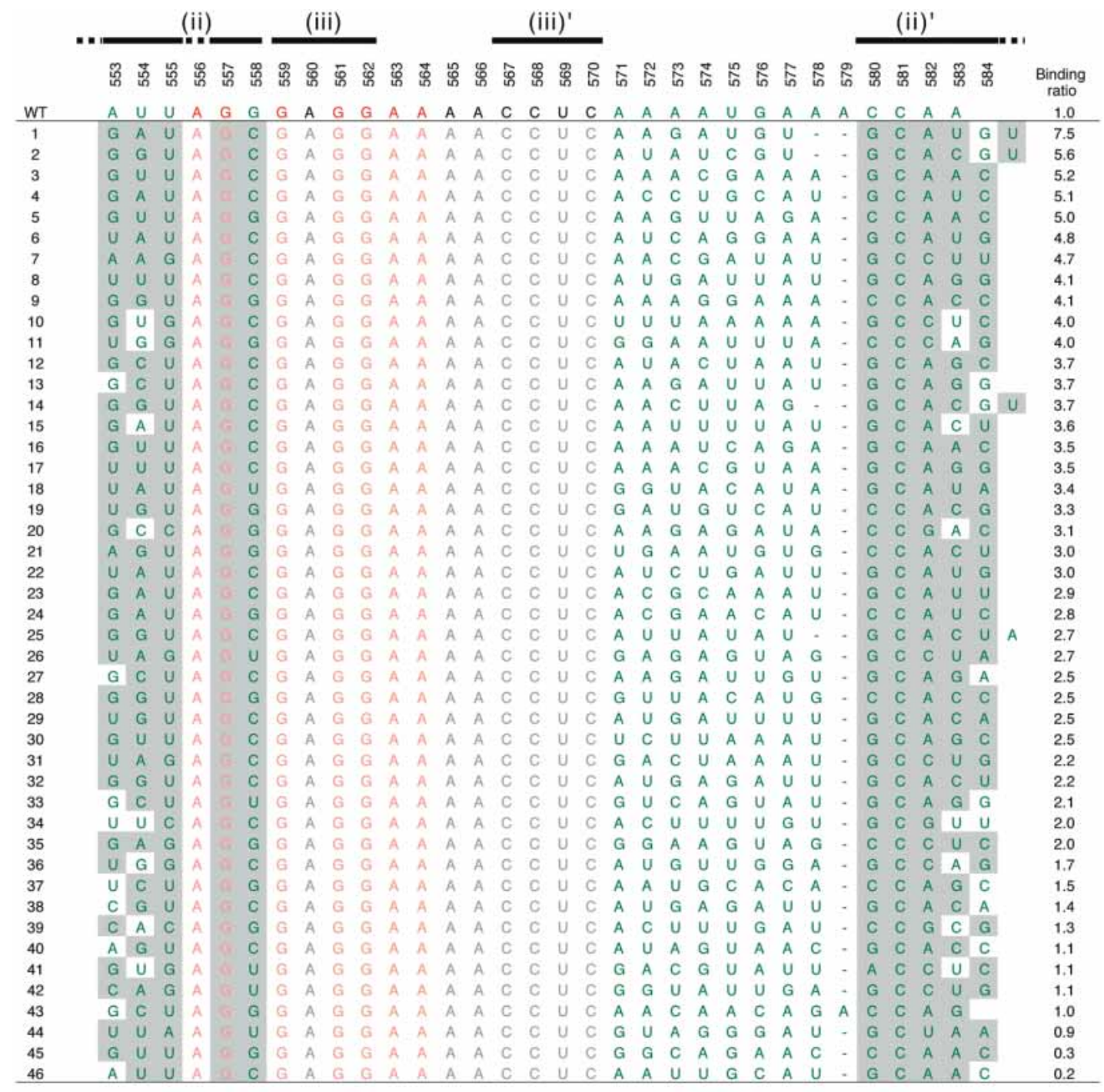

\begin{tabular}{|l|l|llllllllllllllllll|}
\hline \multirow{2}{*}{} & $\mathrm{G}$ & 46 & 25 & 25 & 42 & 33 & 38 & 25 & 50 & 21 & 46 & 42 & 25 & 29 & 33 & 29 & 17 & 21 \\
$\mathrm{8}$ & $\mathrm{A}$ & 21 & 17 & 29 & 21 & 21 & 25 & 21 & 4 & 29 & 17 & 8 & 25 & 29 & 21 & 21 & 38 & 13 \\
$\mathrm{Q}$ & $\mathrm{U}$ & 21 & 25 & 29 & 25 & 25 & 17 & 33 & 25 & 38 & 29 & 17 & 13 & 25 & 29 & 21 & 29 & 33 \\
& $\mathrm{C}$ & 13 & 33 & 17 & 13 & 21 & 21 & 21 & 21 & 13 & 8 & 33 & 38 & 17 & 17 & 29 & 17 & 33 \\
\hline
\end{tabular}

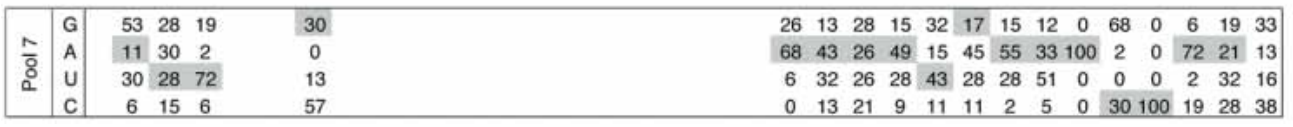

FIGURE 2. Sequences and binding characteristics of DIV+IVa variants obtained by in vitro selection. The figure summarizes RNA sequences inferred from cDNA clones of 46 DIV+IVa variants obtained after seven rounds of in vitro selection for LtrA binding. The wild-type sequence is shown at the top, with the boundaries of predicted secondary structure elements indicated above. Variants 1-46 are listed in order of decreasing binding affinity. Nucleotide residues that were invariant in the in vitro selection of Singh et al. (2002) are shown in red, and those that were randomized in the in vitro selection in this work are shown in green. A dash indicates a gap introduced in the alignment to pair the invariant G557 with a C between positions 579 and 581, resulting in a 1- or 2-nt residue reduction in the size of loop (iii)/(ii) in some variants. Binding ratios determined by competitive binding assays with wild-type DIV+IVa RNA are indicated to the right. Base-paired positions in stem (ii) are highlighted in gray. The bottom shows the percentage of each nucleotide residue at each position in the initial pool (pool 0) and the variants cloned after seven rounds of in vitro selection (pool 7). Numbers highlighted in gray indicate the wild-type nucleotide residue.

bulged A556. Below A556, positions 555:582 are also base paired in all the variants, whereas positions 554:583 are paired in 38 of 46 variants, with seven of the eight at which this position is unpaired compensating by having a GC or CG pair at positions 555:582. Additionally, there was strong selection against a second A residue at position 555 just below A556, and against a $U$ residue between positions 580 and 582. The latter avoids placing a potential base-pairing partner opposite the bulged A556 and also favors strong GC base pairs above A556. 
TABLE 2. Frequencies of base pairs at different positions in the predicted DIVa stem (ii) after in vitro selection for LtrA binding

\begin{tabular}{lrrrrrrrrrr}
\hline & CG & GC & UA & AU & UG & GU & AC & CA & GA & UU \\
\hline $558: 580$ & 27 & 13 & 1 & - & 5 & - & - & - & - & - \\
$557: 581$ & - & $46 *$ & - & - & - & - & - & - & - & - \\
$555: 582$ & 3 & 9 & 33 & 1 & - & - & - & - & - & - \\
$554: 583$ & 6 & 11 & 6 & 12 & 3 & - & 2 & 1 & 2 & 3
\end{tabular}

The table summarizes base-pair frequencies at each position in the predicted DIVa stem (ii) in 46 cloned variants after seven rounds of in vitro selection for LtrA binding. The predicted secondary structures of the variant DIVas are based on an alignment in which the invariant $\mathrm{G} 557$ is base paired with a $\mathrm{C}$ residue between positions 579 and 581 (see text). The resulting GC pair is indicated by an asterisk.

The predicted structure leaves a smaller bulged loop (iii)/ (ii), which in the wild-type sequence contains the AUG initiation codon. Because the invariant G557 base pairs with a $\mathrm{C}$ residue between positions 579 and 581, this loop differs in size in different variants. It has a length of $9 \mathrm{nt}$ in wild type and one variant, $8 \mathrm{nt}$ in 41 variants, and $7 \mathrm{nt}$ in four variants. The two most strongly binding variants (1 and 2) are among the four having the smallest loop (iii)/(ii). Position 571 at the beginning of the loop showed strong selection for a purine residue, whereas positions 577 and 578 within the loop showed selection against a $\mathrm{C}$ residue, possibly to avoid an alternative base-pairing partner for the invariant G557 or other proximate G residues in the stemloop. Mutual information content analysis failed to detect any significant covariation between nucleotide residues in the internal loop (Chiu and Kolodziejczak 1991; Gutell et al. 1992).

Finally, although A553 is bulged in the wild-type sequence, there was strong selection against this feature with only two of the more weakly binding variants (40 and 46) retaining this bulged A. Instead, A553 is replaced by another nucleotide residue in 42 of 46 variants, and the nucleotide residue at position 553 is base paired in 37 of 46 variants, including all of the most tightly binding (1-12). The selection against the bulged A553 present in the wild-type structure could reflect that it decreases the stability of the stem and/or interferes with recognition of the bulged A556, which may be critical for LtrA binding.

\section{Effect of mutations in the bulged $A$ residues on LtrA binding}

To test the importance of the two bulged A residues for LtrA binding, we made mutant DIV+IVa constructs in which each of these A residues was either deleted or paired with a $U$ residue inserted on the opposite side of the stem. The mutant DIV+IVa RNAs were then tested for their ability to bind LtrA by a competition binding assay with an electrophoretically distinguishable wild-type RNA. As shown in Figure 3, mutations that deleted or paired the bulged A556 ( $\Delta$ A556 and A556:U, respectively) strongly decreased the binding affinity for LtrA (binding ratios 0.05 and 0.04 , respectively). By contrast, mutations that deleted or paired the bulged A553 ( $\Delta 553$ and A553:U) increased the binding affinity for LtrA (binding ratios 2.5 and 2.8, respectively), as expected from the selection against this feature. These findings demonstrate that the bulged A556 is important for LtrA binding and that the bulged A553 antagonizes LtrA binding.

\section{Effect of bulged A mutations on RNA splicing in vivo}

We next tested whether mutations in the bulged A residues affect maturase-promoted splicing in vivo. For this purpose,

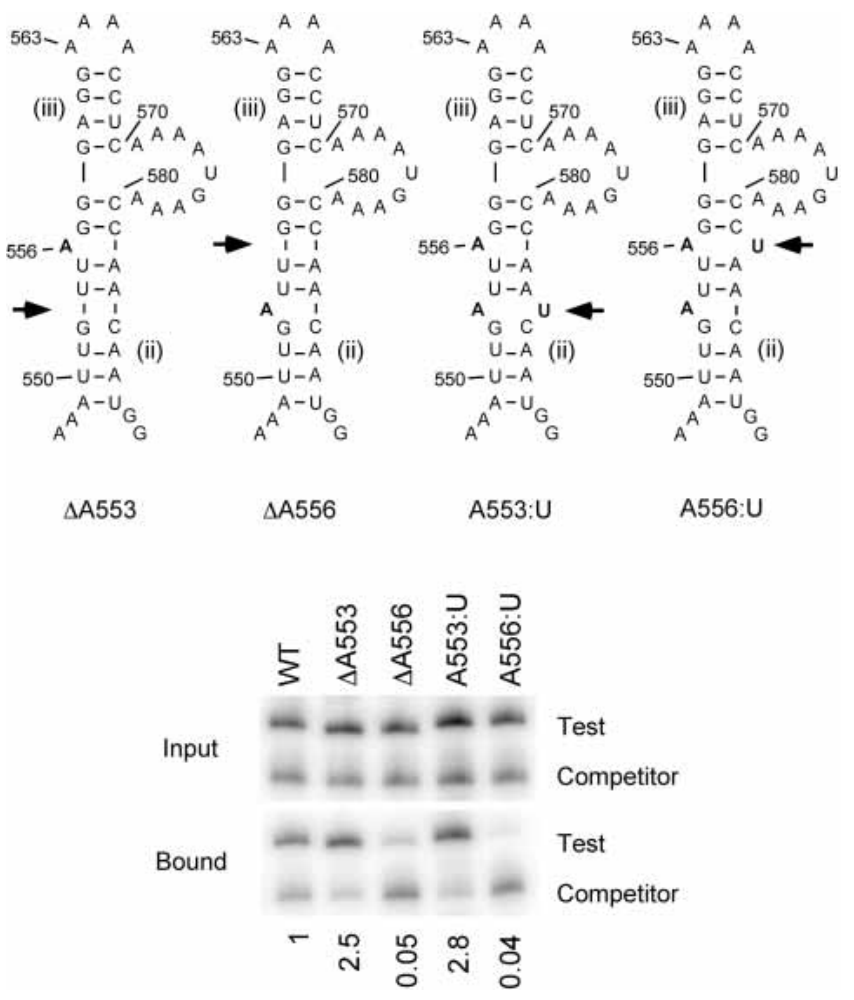

FIGURE 3. Effect of mutations in the bulged A556 and A553 residues on LtrA binding. The top shows the predicted secondary structure of mutant DIV+IVa RNAs in which A556 and A553 were either deleted or paired with a $U$ residue inserted on the opposite side of the stem. The bulged A residues and complementary $U$ residues are shown in bold, and the site of mutation in each variant is indicated by an arrow. Competitive binding assays with ${ }^{32} \mathrm{P}$-labeled mutant (test) and wildtype (competitor) RNAs were carried out by incubating a mixture of $100 \mathrm{nM}$ of each RNA with a limiting amount of LtrA protein $(10 \mathrm{nM})$ for $60 \mathrm{~min}$ at $30^{\circ} \mathrm{C}$, and then filtering through nitrocellulose to bind RNA/protein complexes. Input RNAs and bound RNAs eluted from the nitrocellulose filter after LtrA binding were analyzed in a denaturing $5 \%$ polyacrylamide gel. To correct for changes in binding affinity due to differences in flanking sequences, binding ratios were normalized based on a parallel competition assay between two wildtype DIV+IVa RNAs having the same flanking sequences (left lane; see Materials and Methods). A repeat of the experiment gave the same results. 
A

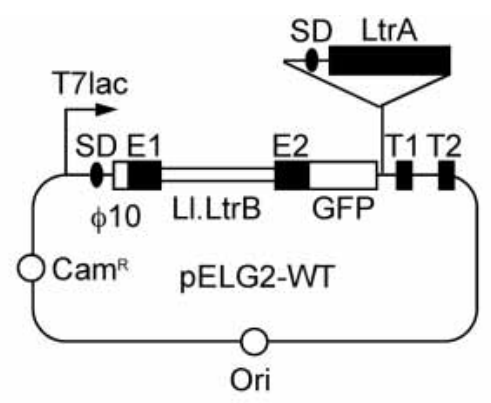

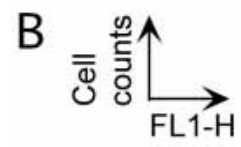
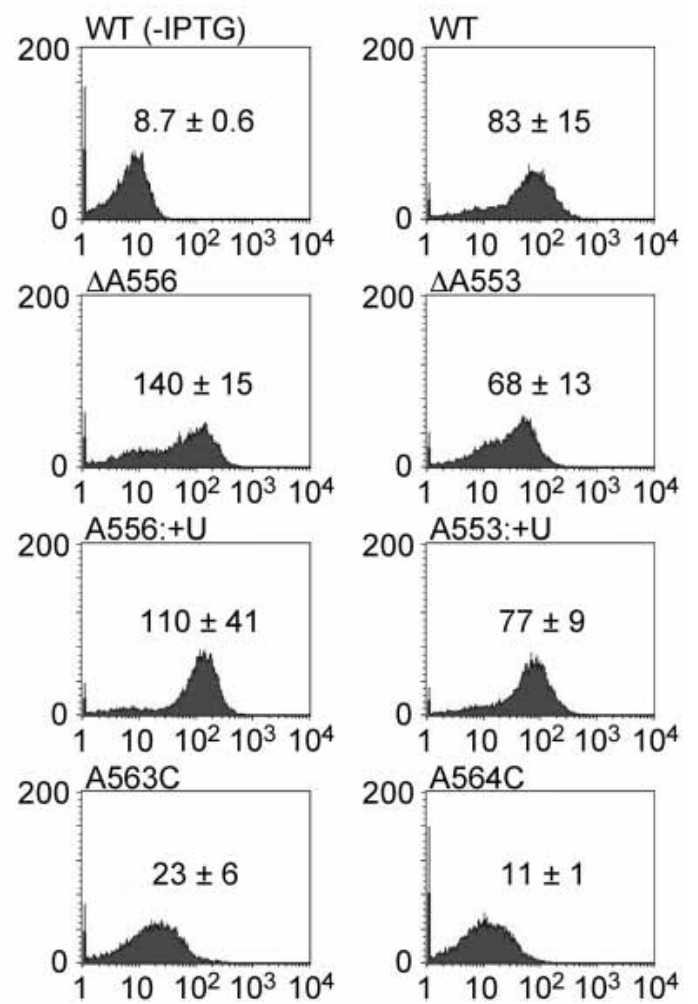

FIGURE 4. Effect of DIV+IVa mutations on in vivo splicing of the Ll.LtrB- $\triangle$ ORF intron. (A) Plasmid pELG2-WT contains an $l$ trB/GFP fusion, which links the splicing of a 0.9-kb Ll.LtrB- $\Delta$ ORF intron to the expression of GFP. The 1 trB/GFP fusion is cloned downstream from a T7lac promoter in a pET-11a-based plasmid, with the LtrA ORF cloned just downstream from the $3^{\prime}$ exon/GFP sequence. (SD) The Shine-Dalgarno sequence preceding the LtrA ORF. (B) FACS assays. pELG2 plasmids containing the wild-type Ll.LtrB- $\Delta$ ORF or indicated mutant introns were transformed into E. coli HMS174(DE3), and cells with or without (-IPTG) induction were analyzed by using a FACS. The figure shows a set of representative FACS assays, with the value in each panel indicating the average peak position \pm the standard deviation in three independent experiments.

we used plasmid pELG2, which contains an ltrB/GFP fusion in which the splicing of a $0.9-\mathrm{kb} \triangle \mathrm{ORF}$ derivative of the Ll.LtrB-intron is linked to the expression of GFP (Fig. 4A; Cui et al. 2004). The $l t r B / G F P$ fusion is cloned downstream from a phage T7lac promoter, and the LtrA protein pre- ceded by a Shine-Dalgarno sequence is expressed from a position just downstream from the GFP coding sequence. This arrangement makes it possible to introduce mutations into DIVa of the Ll.LtrB- $\Delta$ ORF intron without affecting the expression of LtrA. Splicing was assayed in Escherichia coli HMS174(DE3), which contains an integrated isopropylthio- $\beta$-D-galactoside (IPTG)-inducible T7 RNA polymerase, by measuring single-cell fluorescence using a fluorescence-activated cell sorter (FACS). The FACS assay, which measures production of spliced product, was shown previously to give results in close agreement with both RT-PCR and poison primer extension assays of in vivo splicing (Cui et al. 2004).

As shown in Figure 4B, IPTG induction of cells containing the wild-type construct resulted in an almost 10-fold increase in GFP fluorescence indicative of in vivo splicing ( $83 \pm 15$ units compared to $8.7 \pm 0.6$ units with and without IPTG induction, respectively). The deletion or pairing of A556, which results in weaker binding of LtrA, appeared to somewhat increase the level of in vivo splicing $(140 \pm 15$ and $110 \pm 41$ units, respectively), whereas the deletion or pairing of A553, which results in stronger binding of LtrA, appeared to slightly decrease the level of in vivo splicing (68 \pm 13 and $77 \pm 9$ units, respectively). Although these effects are relatively small, the same trends were observed for mutations in both bulged A residues in poison primer extension assays of in vivo splicing (Fig. 5). By comparison, the mutations $\mathrm{A} 563 \mathrm{C}$ and $\mathrm{A} 564 \mathrm{C}$ in the two invariant $\mathrm{A}$
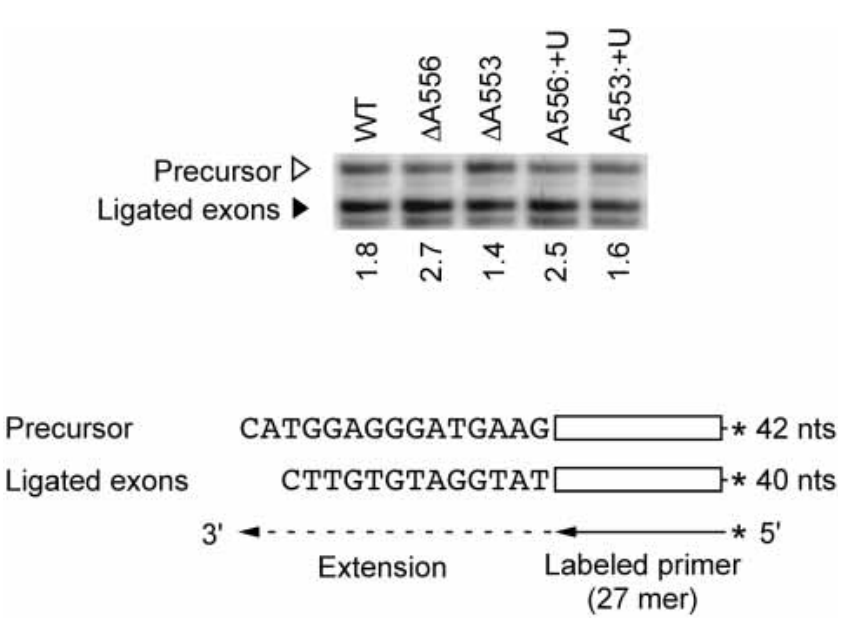

FIGURE 5. Poisoned primer extension assay of in vivo RNA splicing. RNAs from cells containing pELG2-WT or the indicated DIVa mutants were used for poisoned primer extension with M-MLV RT and a $5^{\prime}{ }^{32} \mathrm{P}$-labeled primer complementary to exon 2 (E2). In the presence of $\mathrm{ddC}$, primer extension stops after the first $\mathrm{G}$ residue in the template, resulting in primer extension products of 42 and $40 \mathrm{nt}$ for unspliced precursor and ligated-exon RNAs, respectively. The products were analyzed in a denaturing $10 \%$ polyacrylamide gel, which was dried and quantified with a PhosphorImager. The ratio of ligated exon to unspliced precursor products is indicted beneath each gel lane. The schematic at the bottom shows the sequence of predicted products for unspliced precursor and ligated-exon RNAs. $\left(^{*}\right) 5^{\prime}$ label. A repeat of the experiment gave the same results. 
residues in loop (iii), which were shown previously to inhibit LtrA binding (Singh et al. 2002), more strongly inhibited splicing (23 \pm 6 and $11 \pm 1$ units, respectively; Fig. 4), and the complete deletion of DIVa was shown previously to inhibit in vivo splicing in this assay by $90 \%-94 \%$ (Cui et al. 2004). The larger effects of the loop (iii) mutations could reflect that they result in a greater decrease in the binding affinity for LtrA than do the bulged A mutations. The larger effects could also reflect that the loop nucleotide residues are more critical than those in the stem for positioning LtrA to interact with the catalytic core, or that the loop and deletion mutations have a greater effect on the underlying ribozyme activity of the intron RNA.

\section{Effect of bulged A mutations on intron mobility}

The effect of the bulged A mutations on intron mobility was tested by using a previously described E. coli two-plasmid assay in which an Ll.LtrB- $\Delta \mathrm{ORF}$ intron with a phage T7 promoter inserted near its $3^{\prime}$ end is expressed from a donor plasmid and integrates into a recipient plasmid target site cloned upstream of a promoterless $t e t^{\mathrm{R}}$ gene, thereby activating that gene (Guo et al. 2000). The intron donor plasmid pACD2 used in these experiments expresses the LtrA ORF from a position downstream from the $3^{\prime}$ exon, again enabling us to introduce mutations into the DIVa structure within the intron without affecting the expression of LtrA (Karberg et al. 2001).

As summarized in Table 3, the deletion or pairing of the bulged A556, which results in weaker binding of LtrA, significantly inhibited intron mobility (mobility frequencies $27 \pm 3.5$ and $9.3 \pm 2.6 \%$ wild type for $\Delta \mathrm{A} 556$ and $\mathrm{A} 556: \mathrm{U}$, respectively). Surprisingly, however, the deletion or pairing

TABLE 3. Effect of DIVa mutations on intron mobility

\begin{tabular}{lc}
\hline Intron & Mobility frequency $(\%)$ \\
\hline WT & 100 \\
$\Delta$ A556 & $27 \pm 3.5$ \\
A556:U & $9.3 \pm 2.6$ \\
$\Delta$ A553 & $25 \pm 3.7$ \\
A553:U & $50 \pm 14$ \\
A563C & $26 \pm 9.8$ \\
A564C & $1.5 \pm 1.4$
\end{tabular}

Mobility frequencies were determined by using an E. coli twoplasmid assay. The $\mathrm{Cam}^{\mathrm{R}}$ intron-donor plasmid $\mathrm{pACD} 2$ expresses an LI.LtrB- $\Delta$ ORF intron with a phage T7 promoter near its 3' end, with the LtrA protein expressed from a position downstream of the $3^{\prime}$ exon. The $A m p^{R}$ recipient plasmid pBRR3-ItrB contains the LI.LtrB target site (positions -30 to +15 ) cloned upstream of a promoterless $t e t^{R}$ gene, so that insertion of the intron into the target site activates the expression of the tet $t^{R}$ gene. Mobility frequencies are defined as the ratio of $\left(\mathrm{Tet}^{\mathrm{R}}+A m p^{R}\right) / \mathrm{Amp}^{\mathrm{R}}$ colonies, normalized to that of the wild-type LI.LtrB- $\Delta$ ORF intron assayed in parallel. The values shown are the mean \pm the standard deviation for three independent experiments. The mobility frequencies of the wildtype LI.LtrB intron in these experiments ranged from 38 to $52 \%$. of the bulged A553, which results in tighter binding to DIVa RNAs, also inhibited intron mobility (mobility frequencies $25 \pm 3.7$ and $50 \pm 14 \%$ wild type for $\triangle \mathrm{A} 553$ and $\mathrm{A} 553: \mathrm{U}$, respectively), as did the mutations $\mathrm{A} 563 \mathrm{C}$ and $\mathrm{A} 564 \mathrm{C}$ in loop (iii) (mobility frequencies $26 \pm 9.8$ and $1.5 \pm 1.4 \%$ wild type, respectively). By comparison, deletion of the entire DIVa stem-loop reduced the mobility frequency to $6 \times 10^{-6}$ in this assay (D'Souza and Zhong 2002).

\section{DISCUSSION}

Our results show that the high affinity LtrA binding site in DIVa of the Ll.LtrB intron consists of terminal stem loop, a bulged purine-rich loop, and a stem with two bulged A residues. The bulged A556 is required for tight binding of LtrA to DIV+DIVa RNA, whereas the bulged A553 antagonizes this binding. The structure predicted here for the DIVa binding site is supported by previous chemical-modification experiments with the intact Ll.LtrB- $\Delta \mathrm{ORF}$ intron (Matsuura et al. 2001). These experiments showed that the bulged A556, as well as all A and G residues in the two base pairs above and below A556 (G557, G588, A582, and A583) are accessible to dimethylsulfate or kethoxal modification in the free RNA at 5 or $50 \mathrm{mM} \mathrm{Mg}{ }^{2+}$, but strongly protected under these conditions by LtrA binding (Matsuura et al. 2001). Additionally, the protections by LtrA extend throughout stem (ii), with the bulged A553 being moderately protected. By contrast, $A$ and $G$ residues in the predicted purine-rich loop (A570-A579) were accessible in the free RNA at $5 \mathrm{mM} \mathrm{Mg}^{2+}$, moderately protected by RNA structure at $50 \mathrm{mM} \mathrm{Mg}^{2+}$, and not further protected by LtrA binding. Importantly, the modification of the A and G residues in the two base pairs above and below A556 in the free RNA implies that the formation of these base pairs and the consequent bulging of A556 must be stabilized by LtrA.

It is also instructive to compare our results with those of the previous in vitro selection, in which DIVa positions 537-598 were "doped" 30\%. In that selection, both A556 and G557 were invariant in all tightly binding DIVa variants, whereas other nucleotide residues at the top of stem (ii) as drawn here were conserved in most clones but not invariant (Singh et al. 2002). Twenty-nine of 30 variants could be drawn in a configuration similar to that in Figure $1 \mathrm{~B}$ with A556 bulged and two base pairs above A556, whereas the remaining variant had just a single GC pair above the bulged A556. There was more variability in the number of base pairs below A556, and in four variants, a G at position 555 was also bulged before resuming stem (ii). Importantly, A556 was unpaired in all of the variants. The greater diversity of structures in the previous selection presumably reflects that more nucleotide residues were allowed to vary, providing more degrees of freedom compatible with LtrA binding.

The binding of LtrA to DIVa is important for translational regulation, RNA splicing, and intron mobility (see 
Introduction). Our results show that the deletion or pairing of the bulged A556, which is required for tight binding of LtrA to DIV+IVa RNA, had relatively little effect on RNA splicing in vivo (perhaps a slight increase in the level of spliced product), but significantly inhibited intron mobility. Surprisingly, however, the deletion or pairing of the bulged A553, which leads to tighter binding of LtrA, also significantly inhibited mobility. Mutations in the critical nucleotide residues A563 and A564 in the terminal loop (iii), shown previously to inhibit LtrA binding (Singh et al. 2002), inhibited both RNA splicing and intron mobility. Together, these findings show that mutations in different regions of DIVa can differentially affect RNA splicing and intron mobility.

DIVa lies outside the catalytic core, and small mutations in this structure are not expected to strongly affect the ribozyme activity of the intron RNA. The relatively small effects of the DIVa mutations on RNA splicing reflect that LtrA binding to catalytic core regions is more critical for that process than is its binding to DIVa (Cui et al. 2004), whereas the larger effects on intron mobility may reflect that LtrA binding to DIVa is particularly important for positioning the protein for initiation of cDNA synthesis (Wank et al. 1999). Additionally, our results suggest that the binding strength of LtrA to DIVa is delicately tuned, so that either too tight or too weak binding can inhibit mobility. Because LtrA also functions as a translational repressor, too tight binding of LtrA to DIVa could also deleteriously affect translation, particularly if a second round of translation from the same intron is required for synthesis of active LtrA dimers (Cui et al. 2004). These considerations provide an explanation for the persistence of the bulged A553 in the wild-type Ll.LtrB structure, even though its presence impedes LtrA binding.

The LtrA/DIVa interaction characterized here is reminiscent of coat protein/RNA hairpin interactions used for translational repression of viral replicase synthesis in singlestranded RNA phages (Uhlenbeck et al. 1983; Witherell et al. 1991; Draper et al. 1995; Lim and Peabody 2002; Spingola et al. 2002). In these phages, coat protein dimers use a symmetrical $\beta$-sheet formed across the dimer interface to bind an RNA stem-loop that has a bulged A on the $5^{\prime}$ side of the stem. The stem-loop contains the replicase initiation region, which is occluded by coat protein binding to downregulate translation (Draper 1995, 1999). X-ray crystallography of the phage MS2 protein bound to the RNA-stem loop showed that amino acid residues in the $\beta$-sheet recognize specific bases in the loop and the bulged A-residue, which are constrained in a fixed three-dimensional geometry by the stem-loop (Valegard et al. 1994). In other phage, however, the same $\beta$-sheet region has adopted or evolved to recognize divergent stem-loop structures that differ in the size of the loop and stem, and the requirement for bulged nucleotide residues (Draper 1995; Lim and Peabody 2002; Spingola et al. 2002).
The DIVa structures of other group II introns are generally similar to those of the yeast and lactococcal introns in consisting of stem-loops with irregular kinks and bulges (see Singh et al. 2002). The yeast aI1 and aI2 DIVa stemloops, which are discriminated by their respective IEPs, each contains a 6-nt loop, which differs in sequence between the two introns, and the stems also differ in having bulges at different positions (Huang et al. 2003). Our results suggest that key maturase recognition elements in these and other group II intron DIVas may include the terminal-stem loop, as well as bulged nucleotide residues farther down the stem. However, the findings for the phage coat protein/ RNA hairpin interactions suggest that different IEPs could have diverged to recognize somewhat different elements in this common structural framework.

\section{MATERIALS AND METHODS}

\section{Bacterial strains and growth conditions}

E. coli strain HMS174(DE3) was used for in vivo splicing and intron mobility assays, BL21(DE3) was used for the expression of the LtrA protein, and $\mathrm{DH} 5 \alpha$ was used for cloning. Cells were grown in LB medium, except those for LtrA protein expression, which were grown in SOB medium. Antibiotics were added at the following concentrations: ampicillin $(100 \mu \mathrm{g} / \mathrm{mL})$, chloroamphenicol $(25 \mu \mathrm{g} / \mathrm{mL})$, and tetracycline $(25 \mu \mathrm{g} / \mathrm{mL})$.

\section{Recombinant plasmids}

pKW-DIV+IVa and its mutant derivatives, used to synthesize in vitro transcripts for RNA binding experiments, contain the DIV+IVa region of the Ll.LtrB- $\Delta$ ORF intron (positions 526-605/ 2383-2395; the discontinuity in position numbers reflects deletion of the intron ORF) cloned behind a phage T7 promoter in pUC19 (Yanisch-Perron et al. 1985). To construct these plasmids, two PCRs were carried out using as template an Ll.LtrB- $\triangle$ ORF intron deleted for DIVb (positions 606-2382; Wank et al. 1999). One PCR used the primer DIVupL (5' -AGGAAAGCTTTAATACGACT CACTATAGGGCGCAGTCCAACTCACAGGTTATTGTGTACTA AAATTAAAA), which introduces a HindIII site, T7 promoter, and the 20-nt $5^{\prime}$ flanking sequence GGGCGCAGTCCAACTCACAG, in combination with a 30- to 38-nt DNA oligonucleotide primer encoding the mutant DIVa sequence. The other PCR used an overlapping oligonucleotide complementary to the DIVa primer and the primer DIVdnL (5'-TCGTGTGTCTAGACGCGTGAT CAGTTATTGTTCGTTATTCTTTC), which introduces a 10-nt 3' flanking sequence (TGATCACGCG) and an XbaI site. The products of the two PCRs were gel purified, mixed, and amplified with the outside primers pDIVupL and pDIVdnL, and the resulting 162- to 164-bp PCR products, corresponding to the wild-type sequence or mutant sequences with 1-nt deletions or insertions, were gel purified, digested with HindIII and XbaI, and cloned between the corresponding sites of pUC19.

pACD2, the donor plasmid used in intron mobility assays, contains a 0.9-kb Ll.LtrB- $\Delta$ ORF intron and short flanking exons cloned behind a T7lac promoter in a Cam ${ }^{\mathrm{R}}$ pACYC184-derivative 
(Guo et al. 2000; Karberg et al. 2001). The intron contains an additional T7 promoter inserted near the $3^{\prime}$ end of DIV, and the LtrA protein is expressed from a position just downstream from the $3^{\prime}$ exon. The recipient plasmid pBRR3-ltrB contains the Ll.LtrB homing site (positions -30 to +15 relative to the introninsertion site) cloned upstream of a promoterless $t e t^{\mathrm{R}}$ gene in an $\mathrm{Amp}^{\mathrm{R}}$ pBR322-derivative (Guo et al. 2000; Karberg et al. 2001). DIVa mutations were recloned into pACD2 from the pKWDIV+IVa plasmids described above by carrying out PCRs of pACD2 with primers S1 (5'-CACCACATTTGTACAATCTGTAG GAGAACC) plus A1 (5' -CAATTTTTAATTTTAGTACACAATAA CTGTACC) to amplify intron positions 299-552, and with primers S2 (5'-CAATGGCAATTTTAGAAAGAATCAG) plus A2 (5' CATTGCCATTTCCCAACGCGTCGCCAC) to amplify intron positions 584-690/2287-2301. The gel-purified PCR products were then mixed with the appropriate pKW-DIV+IVa plasmid, and PCR was carried out with primers $\mathrm{S} 1$ and A2 to yield a continuous DNA fragment corresponding to Ll.LtrB intron positions 229690/2287-2301. The final gel-purified PCR product was digested with BsrGI and MluI and cloned between the corresponding sites of pACD2.

pELG2, used for in vivo splicing assays, is an Amp ${ }^{\mathrm{R}} \mathrm{pET}-11 \mathrm{a}-$ derivative, which expresses the Ll.LtrB- $\Delta$ ORF intron and short flanking exons, with the $3^{\prime}$ exon fused in frame to GFP, so that splicing of the intron is linked to the expression of GFP (Cui et al. 2004). The $l$ trB/GFP fusion is cloned downstream from a T7lac promoter, with the LtrA protein expressed from a position just downstream from the $3^{\prime}$ exon/GFP sequence. To reclone DIVa mutations into pELG2, the corresponding pACD2-based plasmid was first digested with SalI and religated to delete the internal T7 promoter in DIV, which would otherwise interfere with the analysis, then digested with HindIII and KpnI to yield a 957-bp fragment that was recloned between the corresponding sites of pELG2.

For each construct, the inserted region was sequenced completely to verify that no adventitious mutations had been introduced during PCR.

\section{Preparation of LtrA protein}

The LtrA protein was expressed in E. coli BL21(DE3) from the intein-based expression vector pImp-1P and purified as described (Saldanha et al. 1999). Protein concentrations were determined by Bradford assay (Coomassie Protein Assay Reagent; Pierce), using bovine serum albumin as a standard, with a correction based on a calibrated LtrA preparation whose concentration was determined by $A_{280}$ (Saldanha et al. 1999). LtrA preparations were checked for in vitro splicing activity before use.

\section{In vitro transcription}

In vitro transcription was carried out with phage T7 RNA polymerase (Stratagene) in the manufacturer's buffer with $2.5 \mathrm{mM}$ of each NTP, $10 \mathrm{mM}$ dithiothreitol, $3.75 \mathrm{mM} \mathrm{MgCl}_{2}, 0.28$ unit/ $\mu \mathrm{L}$ RNase inhibitor (Amersham Bioscience) for $3 \mathrm{~h}$ at $30^{\circ} \mathrm{C}$. To synthesize ${ }^{32} \mathrm{P}$-labeled RNAs for competition binding assays, the reaction medium was supplemented with $330 \mathrm{nM}\left[\alpha-{ }^{32} \mathrm{P}\right] \mathrm{UTP}$ (3,000 Ci/mmole; DuPont-New England Nuclear). Following transcription, the DNA template was digested with DNase I (10 units, $15 \mathrm{~min}, 37^{\circ} \mathrm{C}$; Amersham Bioscience), and transcripts were extracted with phenol-CIA (phenol/chloroform/isoamyl alcohol, 25:24:1 by volume), and ethanol precipitated. Transcripts used for in vitro selection were purified by gel filtration twice through Sephadex G25 (Sigma-Aldrich), and those used in the competition binding assays were purified by electrophoresis in a denaturing $6 \%$ polyacrylamide gel.

\section{In vitro selection of DIVa variants}

The initial DIVa pool for in vitro selection was generated by PCR of a 136-nt DNA oligonucleotide, randmDIV (5' -GGGCCAGTCC AACTCACAGGTTATTGTGTACTAAAATTAAAAATTGNNNAG NGAGGAAAACCTCNNNNNNNNNNNNNCAATGGCAATTTT AGAAAGAATAACGAACAATAACTGATCACGCGTCTAGACA CACGA; where $\mathrm{N}$ = indicates synthesis with equimolar concentrations of $\mathrm{G}, \mathrm{A}, \mathrm{T}$, and $\mathrm{C}$ ), with primers DIVup (5'-AGGAAAGC TTTAATACGACTCACTATAGGGCGCAGTCCAACTCACAG) and DIVdn (5'-TCGTGTGTCTAGACGCGTGATCAG). The PCR was carried out in $25100-\mu \mathrm{L}$ reactions, each containing 20 pmoles of the DNA oligonucleotide, 100 pmoles of each primer, $0.25 \mathrm{mM}$ of each dNTP, 2.5 units Taq DNA polymerase (Invitrogen), and buffer supplied by the manufacturer. Thermal cycle conditions were seven cycles of $93^{\circ} \mathrm{C}$ for $30 \mathrm{sec}, 55^{\circ} \mathrm{C}$ for $10 \mathrm{sec}$, and $72^{\circ} \mathrm{C}$ for $30 \mathrm{sec}$, the number of cycles being limited to minimize duplication of DNA sequences. The PCR product was purified in a $1 \%$ agarose gel and transcribed with phage T7 RNA polymerase in the presence of $\left[\alpha-{ }^{32} \mathrm{P}\right]$ UTP to generate the initial RNA pool.

For in vitro selection, 200 pmoles of the ${ }^{32} \mathrm{P}$-labeled RNA pool were dissolved in $20 \mu \mathrm{L}$ of distilled water and denatured by heating to $90^{\circ} \mathrm{C}$ for $1 \mathrm{~min}$ and then quickly cooled by adding $780 \mu \mathrm{L}$ prewarmed $\left(30^{\circ} \mathrm{C}\right)$ renaturation buffer $\left(128 \mathrm{mM} \mathrm{NH}_{4} \mathrm{Cl}, 6.41 \mathrm{mM}\right.$ $\mathrm{MgCl}_{2}, 51.3 \mathrm{mM}$ Tris- $\mathrm{HCl}$ at $\mathrm{pH} 7.5,12.8 \mathrm{mM}$ dithiothreitol, 0.28 unit/ $\mu \mathrm{L}$ RNase inhibitor; Amersham Bioscience). After incubating at $30^{\circ} \mathrm{C}$ for $1 \mathrm{~min}, 100 \mu \mathrm{L} 4 \mathrm{M} \mathrm{NH}_{4} \mathrm{Cl}$ was added to bring the final $\mathrm{NH}_{4} \mathrm{Cl}$ concentration to $500 \mathrm{mM}$, followed by $100 \mu \mathrm{L} \mathrm{LtrA}$ protein ( $1.5 \mu \mathrm{M}$ for the initial round and $0.1 \mu \mathrm{M}$ for subsequent rounds). The solution was incubated for $1 \mathrm{~h}$ at $30^{\circ} \mathrm{C}$, then filtered through a nitrocellulose membrane filter (BA85; Schleicher \& Schuell) to collect RNA-protein complexes. After washing three times with 2 $\mathrm{mL}$ AMT $\left(500 \mathrm{mM} \mathrm{NH}_{4} \mathrm{Cl}, 5 \mathrm{mM} \mathrm{MgCl}_{2}, 40 \mathrm{mM}\right.$ Tris- $\mathrm{HCl}$ at $\mathrm{pH}$ 7.5), RNA was extracted by vortexing the filter in $400 \mu \mathrm{L} 10 \mathrm{mM}$ Tris- $\mathrm{HCl}$ (pH 7.5), $1 \mathrm{mM}$ EDTA plus $400 \mu \mathrm{L}$ phenol-CIA and then incubating for $3 \mathrm{~h}$ at room temperature. The extracted RNA was ethanol precipitated and reverse transcribed using Superscript II RT (Invitrogen) with primer DIVdn, following the manufacturer's protocol, then PCR amplified with primers DIVup and DIVdn to generate the DNA pool for the next round of selection.

\section{Competition binding assays}

Competition binding assays were carried out as described (Saldanha et al. 1996). ${ }^{32} \mathrm{P}$-labeled wild-type and mutant RNAs were transcribed from cloned (individual variants) or PCR-generated DNA templates (RNA pools) and tested against a wild-type DIV+IVa competitor RNA transcribed from a clone with shorter 5' - and 3'-flanking sequences (GGG and TCTAG, respectively). A mixture containing $100 \mathrm{nM}$ of each RNA was incubated with 10 $\mathrm{nM}$ LtrA protein in $100 \mu \mathrm{L}$ AMT containing $5 \mathrm{mM}$ dithiothreitol and 0.28 unit/ $\mu \mathrm{L}$ RNase inhibitor (Amersham Bioscience) for 60 
min at $30^{\circ} \mathrm{C}$, and then filtered through nitrocellulose to bind RNA-protein complexes. The RNAs were recovered from the filter by phenol-CIA extraction, as described above, followed by ethanol precipitation in the presence of $1 / 10$ volume of $3 \mathrm{M}$ ammonium acetate and E. coli tRNA carrier (10-20 $\mu$ g; Sigma-Aldrich), and then analyzed in a denaturing 5\% polyacrylamide gel, which was dried and quantified using a PhosphorImager (model 445SI; Molecular Dynamics). The binding ratio is defined as the ratio of the mutant to wild-type RNA bound by LtrA relative to the input ratio of the RNAs. To control for the effect of different flanking sequences used to generate different-sized transcripts, the binding ratios were normalized based on binding ratios determined in parallel for two wild-type RNAs having the same flanking sequences (1.17 and 1.19 in two experiments).

\section{FACS assay of in vivo RNA splicing}

E. coli HMS174(DE3), which carries an IPTG-inducible T7 RNA polymerase, was transformed with pELG2 plasmids containing wild-type and mutant Ll.LtrB introns, and a single colony was inoculated into $5 \mathrm{~mL} \mathrm{LB}$ medium plus $100 \mu \mathrm{g} / \mathrm{mL}$ ampicillin and grown overnight with shaking at $37^{\circ} \mathrm{C}$. Fifty microliters of the overnight culture were then inoculated into $5 \mathrm{~mL}$ of flesh $\mathrm{LB}$ plus ampicillin, grown at $37^{\circ} \mathrm{C}$ until O.D. ${ }_{595}=0.4$, and induced with $100 \mu \mathrm{M}$ IPTG for $3 \mathrm{~h}$ at $37^{\circ} \mathrm{C}$. Cells were assayed for GFP fluorescence by using a fluorescence-activated cell sorter (FACS Caliber; Becton Dickinson, Immunocytometry Systems), with filter FL1 $(530 \pm 30 \mathrm{~nm})$. The data were analyzed by using the CELLQuest program (Becton Dickinson).

\section{Poisoned primer extension assay of in vivo splicing}

Poisoned primer extension assays (Pikielny and Rosbash 1985) were carried out with whole-cell RNA from E. coli HMS174(DE3) containing pELG2-WT or DIVa mutants and grown and induced with IPTG, as described above for FACS assay. The RNA was extracted as described by Belfort et al. (1990) and further purified by gel filtration (Centri-Sep; Princeton Separations). Five micrograms RNA were annealed with 0.5 pmoles $5^{\prime}$ end-labeled primer 5'-GATTCGTAGAATTAAAAATGATATGGT by incubating in 7 $\mu \mathrm{L} 50 \mathrm{mM}$ Tris- $\mathrm{HCl}$ ( $\mathrm{pH}$ 7.5), $60 \mathrm{mM} \mathrm{NaCl}, 10 \mathrm{mM}$ dithiothreitol for $3 \mathrm{~min}$ at $60^{\circ} \mathrm{C}$, then chilling on ice. After additions to give 125 $\mu \mathrm{M}$ each dATP, dTTP, and dGTP, $500 \mu \mathrm{M}$ ddCTP, and $1.8 \mathrm{mM}$ $\mathrm{MgCl}_{2}$, the reaction was initiated by adding 180 units M-MLV RT (Invitrogen), incubated for $30 \mathrm{~min}$ at $48^{\circ} \mathrm{C}$, and terminated by adding gel loading buffer ( $80 \%$ formamide, $10 \mathrm{mM}$ EDTA at $\mathrm{pH}$ 8.0, xylene cyanol [1 mg/mL], and bromophenol blue $[1 \mathrm{mg} / \mathrm{mL}]$ ). The products were analyzed in a denaturing $10 \%$ polyacrylamide gel, which was dried and quantified with a PhosphorImager.

\section{Intron mobility assays}

Intron mobility frequencies were determined by using an $E$. coli two-plasmid assay in which a $0.9-\mathrm{kb}$ Ll.LtrB- $\Delta \mathrm{ORF}$ intron with a phage T7 promoter inserted near its $3^{\prime}$ end is expressed from a donor plasmid and integrates into a recipient plasmid target site cloned upstream of a promoterless $t e t^{\mathrm{R}}$ gene, thereby activating that gene (Guo et al. 2000; Karberg et al. 2001). The Cap ${ }^{\mathrm{R}}$ intron donor plasmid pACD2 and the $\mathrm{Amp}^{\mathrm{R}}$ recipient plasmid pBRR3ltrB were cotransformed into E. coli HMS174(DE3), and the cells were grown and induced with $100 \mu \mathrm{M}$ IPTG for $1 \mathrm{~h}$ at $37^{\circ} \mathrm{C}$, as described (Guo et al. 2000; Karberg et al. 2001), then plated on LB medium containing ampicillin or ampicillin plus tetracycline. Mobility frequencies are defined as the ratio of $\left(\mathrm{Tet}^{\mathrm{R}}+\mathrm{Amp}^{\mathrm{R}}\right) / \mathrm{Amp}^{\mathrm{R}}$ colonies.

\section{ACKNOWLEDGMENTS}

This work was supported by NIH grant GM37951.

The publication costs of this article were defrayed in part by payment of page charges. This article must therefore be hereby marked "advertisement" in accordance with 18 USC section 1734 solely to indicate this fact.

Received April 29, 2004; accepted June 7, 2004.

\section{REFERENCES}

Belfort, M., Ehrenman, K., and Chandry, P.S. 1990. Genetic and molecular analysis of RNA splicing in Escherichia coli. Methods Enzymol. 181: 521-539.

Belfort, M., Derbyshire, V., Parker, M.M., Cousineau, B., and Lambowitz, A.M. 2002. Mobile introns: Pathways and proteins. In Mobile DNA II (eds. N.L. Craig et al.), pp. 761-783. ASM Press, Washington, DC.

Chiu, D.K. and Kolodziejczak, T. 1991. Inferring consensus structure from nucleic acid sequences. Comput. Appl. Biosci. 7: 347-352.

Cousineau, B., Smith, D., Lawrence-Cavanagh, S., Mueller, J.E., Yang, J., Mills, D., Manias, D., Dunny, G., Lambowitz, A.M., and Belfort, M. 1998. Retrohoming of a bacterial group II intron: Mobility via complete reverse splicing, independent of homologous DNA recombination. Cell 94: 451-462.

Cui, X., Matsuura, M., Wang, Q., Ma, H., and Lambowitz, A.M. 2004. A group II intron-encoded maturase functions preferentially in cis and requires both the reverse transcriptase and $\mathrm{X}$ domains to promote RNA splicing. J. Mol. Biol. 340: 211-231.

D'Souza, L.M. and Zhong, J. 2002. Mutations in the Lactococcus lactis Ll.LtrB group II intron that retain mobility in vivo. BMC Mol. Biol. 3: 17.

Draper, D.E. 1995. Protein-RNA recognition. Annu. Rev. Biochem. 64: 593-620.

Draper, D.E. 1999. Themes in RNA-protein recognition. J. Mol. Biol. 293: 255-270.

Eskes, R., Yang, J., Lambowitz, A.M., and Perlman, P.S. 1997. Mobility of yeast mitochondrial group II introns: Engineering a new site specificity and retrohoming via full reverse splicing. Cell 88: 865874.

Eskes, R., Liu, L., Ma, H., Chao, M.Y., Dickson, L., Lambowitz, A.M., and Perlman, P.S. 2000. Multiple homing pathways used by yeast mitochondrial group II introns. Mol. Cell. Biol. 20: 8432-8446.

Guo, H., Karberg, M., Long, M., Jones 3rd, J.P., Sullenger, B., and Lambowitz, A.M. 2000. Group II introns designed to insert into therapeutically relevant DNA target sites in human cells. Science 289: 452-457.

Gutell, R.R., Power, A., Hertz, G.Z., Putz, E.J., and Stormo, G.D. 1992. Identifying constraints on the higher-order structure of RNA: Continued development and application of comparative sequence analysis methods. Nucleic Acids Res. 20: 5785-5795.

Huang, H.R., Chao, M.Y., Armstrong, B., Wang, Y., Lambowitz, A.M., and Perlman, P.S. 2003. The DIVa maturase binding site in the yeast group II intron aI2 is essential for intron homing but not for in vivo splicing. Mol. Cell. Biol. 23: 8809-8819.

Karberg, M., Guo, H., Zhong, J., Coon, R., Perutka, J., and Lambowitz, A.M. 2001. Group II introns as controllable gene targeting vectors for genetic manipulation of bacteria. Nature Biotechnol. 19: 1162- 
1167.

Lambowitz, A.M. and Zimmerly, S. 2004. Mobile group II introns. Annu. Rev. Genet. (in press).

Lambowitz, A.M., Caprara, M.G., Zimmerly, S., and Perlman, P.S. 1999. Group I and group II ribozymes as RNPs: Clues to the past and guides to the future. In The RNA world, 2nd ed. (eds. R.F. Gesteland et al.), pp. 451-485. Cold Spring Harbor Laboratory Press, Cold Spring Harbor, NY.

Lim, F. and Peabody, D.S. 2002. RNA recognition site of PP7 coat protein. Nucleic Acids Res. 30: 4138-4144.

Matsuura, M., Saldanha, R., Ma, H., Wank, H., Yang, J., Mohr, G., Cavanagh, S., Dunny, G.M., Belfort, M., and Lambowitz, A.M. 1997. A bacterial group II intron encoding reverse transcriptase, maturase, and DNA endonuclease activities: Biochemical demonstration of maturase activity and insertion of new genetic information within the intron. Genes \& Dev. 11: 2910-2924.

Matsuura, M., Noah, J.W., and Lambowitz, A.M. 2001. Mechanism of maturase-promoted group II intron splicing. EMBO J. 20: 72597270.

Pikielny, C.W. and Rosbash, M. 1985. mRNA splicing efficiency in yeast and the contribution of nonconserved sequences. Cell 41: 119-126.

Saldanha, R., Ellington, A., and Lambowitz, A.M. 1996. Analysis of the CYT-18 protein binding site at the junction of stacked helices in a group I intron RNA by quantitative binding assays and in vitro selection. J. Mol. Biol. 261: 23-42.

Saldanha, R., Chen, B., Wank, H., Matsuura, M., Edwards, J., and Lambowitz, A.M. 1999. RNA and protein catalysis in group II intron splicing and mobility reactions using purified components. Biochemistry 38: 9069-9083.

Singh, R.N., Saldanha, R.J., D'Souza, L.M., and Lambowitz, A.M. 2002. Binding of a group II intron-encoded reverse transcriptase/ maturase to its high affinity intron RNA binding site involves sequence-specific recognition and autoregulates translation. J. Mol. Biol. 318: 287-303.

Spingola, M., Lim, F., and Peabody, D.S. 2002. Recognition of diverse RNAs by a single protein structural framework. Arch. Biochem. Biophys. 405: 122-129.

Uhlenbeck, O.C., Carey, J., Romaniuk, P.J., Lowary, P.T., and Beckett, D. 1983. Interaction of R17 coat protein with its RNA binding site for translational repression. J. Biomol. Struct. Dyn. 1: 539-552.

Valegard, K., Murray, J.B., Stockley, P.G., Stonehouse, N.J., and Liljas, L. 1994. Crystal structure of an RNA bacteriophage coat proteinoperator complex. Nature 371: 623-626.

Wank, H., SanFilippo, J., Singh, R.N., Matsuura, M., and Lambowitz, A.M. 1999. A reverse transcriptase/maturase promotes splicing by binding at its own coding segment in a group II intron RNA. Mol. Cell 4: 239-250.

Witherell, G.W., Gott, J.M., and Uhlenbeck, O.C. 1991. Specific interaction between RNA phage coat proteins and RNA. Prog. Nucleic Acid Res. Mol. Biol. 40: 185-220.

Yang, J., Zimmerly, S., Perlman, P.S., and Lambowitz, A.M. 1996. Efficient integration of an intron RNA into double-stranded DNA by reverse splicing. Nature 381: 332-335.

Yanisch-Perron, C., Vieira, J., and Messing, J. 1985. Improved M13 phage cloning vectors and host strains: Nucleotide sequences of the M13mp18 and pUC19 vectors. Gene 33: 103-119.

Zimmerly, S., Guo, H., Eskes, R., Yang, J., Perlman, P.S., and Lambowitz, A.M. 1995a. A group II intron RNA is a catalytic component of a DNA endonuclease involved in intron mobility. Cell 83: 529-538.

Zimmerly, S., Guo, H., Perlman, P.S., and Lambowitz, A.M. 1995b. Group II intron mobility occurs by target DNA-primed reverse transcription. Cell 82: 545-554. 

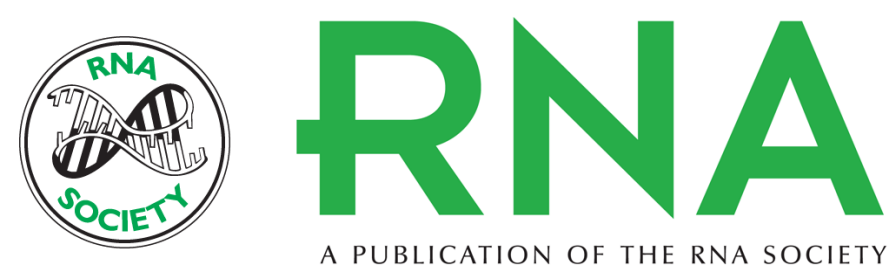

\section{High-affinity binding site for a group II intron-encoded reverse transcriptase/maturase within a stem-loop structure in the intron RNA}

KAZUO WATANABE and ALAN M. LAMBOWITZ

RNA 2004 10: 1433-1443

References This article cites 29 articles, 5 of which can be accessed free at:

http://rnajournal.cshlp.org/content/10/9/1433.full.html\#ref-list-1

\section{License}

Email Alerting

Receive free email alerts when new articles cite this article - sign up in the box at the Service top right corner of the article or click here. 